\title{
Rethinking small molecules for RA
}

Small-molecule inhibitors of mitogenactivated protein kinase (MAPK) have shown great promise as therapeutic agents in preclinical studies of inflammatory arthritis. The disappointing results of the first large, randomized clinical trial of an MAPK inhibitor in patients with rheumatoid arthritis (RA), however, have cast doubt on their efficacy as an oral therapy for the disease. In the study, reported by Stanley Cohen and colleagues in The Journal of Rheumatology, the MAPK pamapimod "was no better than methotrexate in the treatment of RA".

Pamapimod is a novel molecule that selectively inhibits the p38a isoform of MAPK, which is important for the production and signaling of several proinflammatory cytokines involved in the pathogenesis of RA. The agent effectively inhibited tumor necrosis factor, interleukin (IL)-1 and IL-6 production in vivo and in vitro in various models of arthritis. In addition, "animal studies had demonstrated significant improvements in inflammatory arthritis models," Cohen says of pamapimod therapy. The results from several phase I studies, however, did not dispel doubts about the potential benefits of this class of therapeutic agent. "It was that concern that led us to compare this therapy to methotrexate." The investigators chose to compare pamapimod with methotrexate as the latter is the "gold standard" first- line DMARD for RA. "It was felt that if a p38 MAPK was not as good or better than methotrexate in a 'proof-of-concept' study," explains Cohen, "it would not be worthwhile to continue to develop it".

\section{4 ...whether this class of} agents will prove to be a clinically useful therapy for RA remains to be seen 77

To compare the two agents, the investigators randomly assigned 204 patients with RA to receive methotrexate (starting at $7.5 \mathrm{mg}$ per week and escalated to a maximum dose of $20 \mathrm{mg}$ per week) or $50 \mathrm{mg}, 150 \mathrm{mg}$, or $300 \mathrm{mg}$ of pamapimod once-daily. After 12 weeks of treatment, the proportion of patients who fulfilled the American College of Rheumatology criteria for 20\% improvement (ACR20) was significantly higher among those who received methotrexate $(45 \%)$ than those who received pamapimod (23\%, $18 \%$ and $31 \%$ for the $50 \mathrm{mg}, 150 \mathrm{mg}$ and $300 \mathrm{mg}$ doses, respectively). In addition, each of the ACR core criteria improved more from baseline in patients receiving methotrexate in comparison with those receiving any dose of pamapimod. The highest dose of pamapimod (300 mg) improved the number of swollen and tender joints almost as well as did methotrexate, but methotrexate still had a more pronounced effect on disease activity and pain. Adverse events occurred more frequently with pamapimod than with methotrexate, and the frequency increased with increasing dose. The profile of adverse events for pamapimod, which included dizziness, skin disorders and elevated levels of liver enzymes, was similar to that reported with other p38 inhibitors.

"Based on the results of this study," says Cohen, "[pamapimod] is not being carried forward in development". Clinical studies of other p38 inhibitors are underway, but whether this class of agents will prove to be a clinically useful therapy for RA remains to be seen. The development of small-molecule inhibitors is still of great interest, as they could provide a less-expensive, orally administered alternative to parenteral biologic agents. Cohen likens the development of small-molecule agents that have the benefits of parenteral cytokine inhibitors to the "holy grail" of RA therapy. For now, at least, the search continues.

Sarah Price

Original article Cohen, S. B. et al. Evaluation of the efficacy and safety of pamapimod, a p38 MAP kinase inhibitor, in a double-blind, methotrexate-controlled study of patients with active rheumatoid arthritis. Arthritis Rheum. 60, 335-344 (2009). 